\title{
Analysis on Aircraft Sortie Generation Rate Based on Multi-class Closed Queueing Network
}

\author{
ZHENG Mao \\ College of Ship Building Engineering \\ Harbin Engineering University \\ Harbin,150001 ,P.R.China \\ zhmao1987@126.com \\ WANG Chao \\ College of Ship Building Engineering \\ Harbin Engineering University \\ Harbin, 150001 , P.R.China \\ wangchao@hrbeu.edu.cn
}

\author{
WANG Yu-juan \\ College of Ship-building Engineering \\ Harbin Engineering University, HEU \\ Harbin, China \\ yj020111@163.com \\ HUANG Sheng \\ College of Ship-building Engineering \\ Harbin Engineering University, HEU \\ Harbin, China \\ huangsheng@hrbeu.edu.cn
}

\begin{abstract}
In order to research the sortie generation rate (SGR) of carrier-based aircrafts, an analytical method based on closed queueing network was put forward. A multi-class, multi-server non-preemptive (or Head of Line, HOL) closed queueing network model was analyzed. As the exact mathematical solution is not available, an approximate method based on reduced work-load assumption and mean value analysis (MVA) iteration was used to gain the performance of the network. As a result, this approximation method can provide the marginal distribution of customers at each service facility of the queueing network. Numerical results that show that the accuracy of the method is good was given.
\end{abstract}

\section{Keywords-SGR; closed queueing network; HOL; MVA}

\section{INTRODUCTION}

The sortie generation rate (SGR) of carrier-borne crafts is the key component of aircraft carrier's ability to project power against any enemy.In order to gain insight into SGR, queueing theory based on Fork-join closed queueing network have been used ${ }^{[1]}$.Share-service facilities closed queueing network has been also used to gain useful conclusion ${ }^{[2]}$.More over, analysts have historically employed simulation tools, such as LOCM, to analyses carrier-borne crafts' SGR ${ }^{[3]}$.

Unfortunately, most methods were used to analyses the operational concepts that there is only one kind of aircrafts in the queueing network. To enhance the firepower capacity of an aircraft carrier, early-warning aircrafts will be launched, recovered, repaired and maintenanced in priority. This paper presents an analytical approach that produces excellent approximate results for a sortie generation system modeled as a multi-class multi-server non-preemptive closed queueing network and Fork-join nodes. However, the exact solution of this model is not available. Modified-vacation intervals theory was used to get approximate solution $^{[4]}$. Mean value analysis (MVA) is the method which was used to solve multi-class priority open and closed queueing networks ${ }^{[5,6,7]}$. Reiser's work on analysis of Reduced-work rate assumption was proven as effective and accurate ${ }^{[8]}$. In order to gain the SGR of carrier-borne aircrafts, this paper put forward an approximation method based on reduced-work rate assumption, $\mathrm{M} / \mathrm{M} / \mathrm{C}$ non-preemptive queueing theory and Markov network queueing theory. The quality of the analytical approximation is demonstrated through comparision with simulation results.

\section{SGR MODEL DISCRIPTION}

A carrier-borne aircrafts' sortie generation system can be quite complex, a relatively simple queueing model can capture the fundamental process behavior. An aircraft is assumed to operate in a closed loop system and represented by customers of the queuing network and launching, sortie(flight), turnaround, recovery, repairing and maintaining can be represented by $\mathrm{M}$ index stations in a closed queueing network. Let $\mathrm{R}$ be the number of total classes in the network and let Class-0 have the highest priority while Class-R have the lowest priority. Let $N_{r}$ be the total number of class-r customers and $\mathbf{N}=\left(N_{0}, N_{1}, \cdots, N_{R}\right)$ be the population vector. Let $\mathbf{C}=\left(C_{0}, C_{1}, \cdots, C_{R}\right)$ be the number of servers at each station. Each aircraft proceeds to station $\mathrm{j}$ with time-invariant probability $p_{i j}$ which consist the routing matrix $\mathbf{P}=\left[p_{i j}\right]_{M \times M}$.

Assume recovery station is the start of the closed queueing network and each aircraft begins with recovery. With probability $p_{12}$, at least one malfunction is detected and the aircraft receives checking and proceeds to repairing station. Aircrafts without malfunction proceeds to maintaining station with the probability $p_{14}$. After maintaining, each aircraft awaits turnaround operations. Then, Each aircraft awaits launching at catapult A (or catapult $\mathrm{B}$ ) with the probability $p_{56}$ (or $p_{57}$ ) and next cycle begins. Figure 1 illustrates the proceedings of aircrafts between stations in a typical sortie cycle.

Let $\mu_{m r}$ be the mean service rate of each server for a class-r customer at station $\mathrm{m}$. The service time $S_{m r}$ for 
class-r customers at station $\mathrm{m}$ is independent and given by an exponentially distributed random variable with mean $1 / \mu_{m r}$. The service mechanism is FIFO for customers in the same class and HOL for customers in different classes. For each class of customers, the routing matrix is the same. Let $\lambda_{m r}(\mathbf{N}) \quad, \quad L_{\text {system } m r}(\mathbf{N}) \quad, \quad R_{z m r}(\mathbf{N}) \quad$ and $\rho_{m r}(\mathbf{N})=\lambda_{m r}(\mathbf{N}) \cdot S_{m r}$ denote the mean throughput, queueing length, residence time and utilization of class-r customers at station $\mathrm{m}$ when the closed network population is $\mathbf{N}$. Let $P_{m r}(i \mid \mathbf{N})$ be the probability that $i$ class-r customers are at station $\mathrm{m}$ given population vector $\mathbf{N}$.

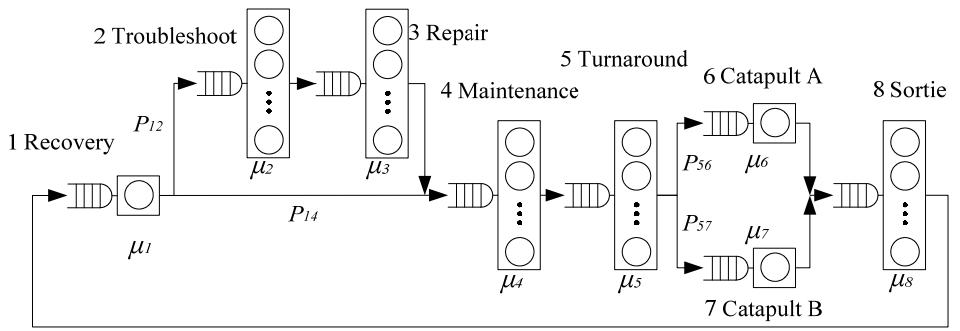

Fig.I Illustration of the CQN system of Aircraft sorties

\section{APPROXIMATIONS FOR PRIORITY QUEUEING NETWORK}

The idea of the method is to calculate the behaviors of each class of customers by using single-class mean value analysis theory. Assume that there are just class-r of customers in the system and other classes of customers can reduce the service rate of class-r customers. So, R equivalent single-class network should be analyzed. The service time and the waiting time caused by serving customers of other classes can be represented as exponentially distributed random variables. The service time will be determined by some assumptions in this paper.

\section{A. The service time of the highest priority class}

When a customer of the highest priority class proceeds to station $m$, it will be served immediately if there are idle servers, otherwise, it should await until any server in station $m$ completes work. The mean proportion of servers which serves class-r at station $m$ is

$$
\Gamma_{m r}(\mathbf{N})=\rho_{m r}(\mathbf{N}) / \sum_{s=0}^{R} \rho_{m s}(\mathbf{N})
$$

The mean time waiting for one server completing its work when all servers at station $m$ are busy can be determined as

$$
\bar{S}_{e m}(\mathbf{N})=\sum_{r=0}^{R} \rho_{m r}(\mathbf{N}) \cdot S_{m r}(\mathbf{N}) / C_{m}
$$

By applying Erlang- $C$ formula, the probability that all servers at station $m$ are busy when one class-r customer less can then be approximately computed as

$$
\begin{aligned}
& P_{\text {BUSY }}\left(n_{r}-1 \mid \mathbf{N}-\mathbf{e}_{r}\right)= \\
& 1-\sum_{\operatorname{sum}\left(n_{r}\right)<C_{m}}\left[P_{m r}\left(n_{r}-1 \mid \mathbf{N}-\mathbf{e}_{r}\right) \prod_{j=0 \& j \neq r}^{R} P_{m j}\left(n_{j} \mid \mathbf{N}\right)\right], r \in R
\end{aligned}
$$

To reduce the complexity of the algorithm, equation (3) calculates the probability that there is at least one idle server. And then, $P_{B U S Y}(\mathbf{N})$ can be gained. Using (1) (3), the modified service time of class- 0 can be detected as equation (4), consisted by 2 parts that one part is class- 0 own service time $S_{m 0}(\mathbf{N})$ and the other part is the time waiting for at least one server completing its work, with a customer of other classes leaving.

$$
\begin{aligned}
& \tilde{S}_{m 0}(\mathbf{N})= \\
& S_{m 0}(\mathbf{N})+P_{B U S Y m}\left(n_{r}-1 \mid \mathbf{N}-\mathbf{e}_{r}\right) \cdot\left[1-\Gamma_{m 0}(\mathbf{N})\right] \cdot \bar{S}_{e m}(\mathbf{N})
\end{aligned}
$$

\section{$B$. The service time of lower priority classes}

When any customer of class-r (class- 0 is not included) proceeds to station $\mathrm{m}$, it is necessary to wait for the customers with the same and even higher priority completing its service. When a customer of class-r waiting for service in the queue at station m, customers with higher priority can arrive and jump the queue (HOL customers). By taking the time waiting for customers of other classes into the service time of class-r, the modified service time of class-r can then be analyzed in 3 parts:

1) The real service time $S_{m r}(\mathbf{N})=1 / \mu_{m r}$

2) The time waiting for customers with higher priority completing service

Let $L_{q m i}(\mathbf{N})$ and $N_{H m r i}(\mathbf{N})$ be the queue length of class-i and the number of class-i HOL customers when a class-r customer waiting in the queue, Then

$$
\begin{aligned}
& L_{q m i}(\mathbf{N})= \\
& \sum_{j=C_{m}}^{N_{r}-1}\left(j-C_{m}+1\right) \cdot P_{m i}\left(j \mid \mathbf{N}-\mathbf{e}_{r}\right) \text {, s.t. } 0 \leq i<r \\
& N_{H m r i}(\mathbf{N})= \\
& {\left[R_{z m r}(\mathbf{N})-S_{m r}(\mathbf{N})\right] \lambda_{m i}(\mathbf{N}) \text {, s.t. } r>0 \quad 0 \leq i<r}
\end{aligned}
$$

Using (3),(5) and (6), the mean time waiting for all the customers with higher priority completing service can be given by

$$
\begin{aligned}
& T_{H ~ m r}(\mathbf{N})= \\
& \sum_{i=0}^{r-1}\left\{P_{\text {BUSY } m}\left(n_{r}-1 \mid \mathbf{N}-\mathbf{e}_{r}\right) \cdot \Gamma_{m i}(\mathbf{N}) \cdot \bar{S}_{e m}(\mathbf{N}) \cdot\left[L_{q m i}(\mathbf{N})+N_{H m r i}(\mathbf{N})\right]\right\}
\end{aligned}
$$


3) The mean time waiting for at least one server completing work without idle servers is obtained as

$$
\begin{aligned}
& T_{1 m r}(\mathbf{N})= \\
& P_{\text {BUSY }}\left(n_{r}-1 \mid \mathbf{N}-\mathbf{e}_{r}\right) \cdot\left[1-\Gamma_{m r}(\mathbf{N})\right] \cdot \bar{S}_{e m}(\mathbf{N})
\end{aligned}
$$

Then, the modified service time of class-r (with lower priority) can be calculated as

$$
\begin{aligned}
& \tilde{S}_{m r}(\mathbf{N})=S_{m r}(\mathbf{N})+T_{1 m r}(\mathbf{N})+T_{H m r}(\mathbf{N})= \\
& S_{m r}(\mathbf{N})+P_{\text {BUSY }}\left(n_{r}-1 \mid \mathbf{N}-\mathbf{e}_{r}\right) \cdot\left[1-\Gamma_{m r}(\mathbf{N})\right] \cdot \bar{S}_{e m}(\mathbf{N})+ \\
& \sum_{i=0}^{r-1}\left\{P_{\text {BUSY }}\left(n_{r}-1 \mid \mathbf{N}-\mathbf{e}_{r}\right) \cdot \Gamma_{m i}(\mathbf{N}) \cdot \bar{S}_{e m}(\mathbf{N}) \cdot\left[L_{q m i}(\mathbf{N})+N_{H m r i}(\mathbf{N})\right]\right\}
\end{aligned}
$$

The modified service time can be represented as Erlang-distributed random variables which have the mean value as the sum of exponentially distributed random variable $^{[6,10,11]}$. the modified service time can be represented as exponentially distributed random variable with the same mean values with the whole delay time ${ }^{[6]}$.

\section{Iteration algorithm}

Because the number of HOL customers is determined by the waiting time of class-r and the throughputs of higher priority classes, it is not available. To gain the number of HOL customers, iteration algorithm is used. The population vector is $\mathbf{N}$ and drop the sign (N) from $\mathbf{P}_{\text {BUSY }}(\mathbf{N}), R_{z m r}^{\Omega}(\mathbf{N}), \lambda_{m r}^{\Omega}(\mathbf{N}), \quad L_{\text {system } m r}^{\Omega}(\mathbf{N}), L_{q m r}^{\Omega}(\mathbf{N})$ and $P_{m r}^{\Omega}(\mathbf{N})$.Let $\Omega$ be the number of iterations.

\section{ALGORITHM:}

Step1 Initialize: $\Omega=1, \overrightarrow{\mathrm{P}}_{\mathrm{BuSY}}^{\Omega=1}=0.1 \cdot \mathbf{e}_{1 \mathrm{MM}}$ (e is a 1 by $M$ matrix of ones, 0.1 is an initial value of $\left.P_{\text {BUSY }}\right), \mathbf{N}_{H}^{\Omega=1}=\mathbf{0}_{\text {mxxxi }}$, $\lambda^{\Omega=1}=\mathbf{e}_{m \times r}, \mathbf{L}_{q}{ }^{\Omega=1}=\mathbf{0}_{m \times r} ;$

Step2 Calculate the modified service time $\tilde{S}_{m r}^{\Omega}$ of class-r at each station with the use of equation (4) or (9);

Step3 The results of Step2 can be used in MVA method. Then, $R_{z m r}^{\Omega}, \lambda_{m r}^{\Omega}, L_{s y s t e m ~ m r}^{\Omega}, L_{q m r}^{\Omega}$ and $P_{m r}^{\Omega}$ of class-r can be calculated with the use of MVA method;

Step4 Calculate the probability, $\overrightarrow{\mathbf{P}}_{\text {BusY }}^{\Omega}$, that all servers in station $m$ are busy by using equation (3).

Step5 Derive the number of HOL customers $N_{H \text { mri }}^{\Omega}$ with the use of (6) and update the modified service time $\tilde{S}_{m r}^{\Omega+1}, \Omega \leftarrow \Omega+1$;

Step6 Determine if the iteration achieves convergence. If it is convergent, Stop the algorithm and output the results. If it does not achieve convergence, go to Step 2.

\section{Simulation of SGR queueing network}

The approximate method (analytical method) in the previous section has been implemented on a personal computer with a Matlab environment. To check the results of analytical method, the closed queueing network is simulated by a Matlab program based on Moute Carlo method. Because of random variation in simulation output, multi replications are used to improve the accuracy. The $95 \%$ confidence interval widths for simulation results, such as queue length and throughput, are also given in Table 2. The simulation analysis requires 11hours.

\section{EXAMPLE}

\section{A. Parameters of the model}

Assume that 2 classes of aircrafts, early warning aircrafts and strickers, are operated on aircraft carrier, and the priority of early warning aircrafts is higher than that of strickers. The routine matrix $\mathbf{P}$, the service rates of each server and the numbers of servers at each station can be

\begin{tabular}{|c|c|c|c|c|c|}
\hline $\begin{array}{c}\text { Server } \\
\text { Stations }\end{array}$ & $\begin{array}{c}\text { Service } \\
\text { Rate } \mu_{i}\end{array}$ & $\begin{array}{l}\text { Number of } \\
\text { servers } C_{i}\end{array}$ & $\begin{array}{c}\text { Server } \\
\text { Stations }\end{array}$ & $\begin{array}{l}\text { Service } \\
\text { Rate } \mu_{i}\end{array}$ & $\begin{array}{l}\text { Number of } \\
\text { servers } C_{i}\end{array}$ \\
\hline Recovery & 50 & 1 & Turnaroud & 10 & 4 \\
\hline Troubleshoot & 20 & $\infty$ & Catapult A & 30 & 1 \\
\hline Repair & 0.56 & 5 & Catapult B & 30 & 1 \\
\hline Maintenance & 1.43 & 5 & Sortie & 0.667 & $\infty$ \\
\hline
\end{tabular}
given in Table 1. Note that, station 2 and 8 are IS stations.

The routine matrix $\mathbf{P}$ is:

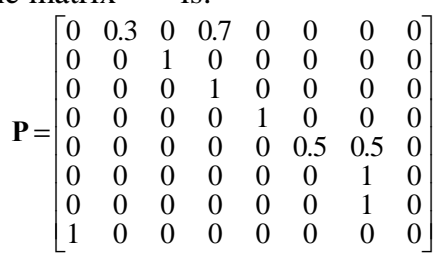

Table I Parameters of closed queueing network

For the Base Case of a foreign aircraft carrier, 2 early warning aircrafts and 36 strickers are operated. Then, the population vector $\overrightarrow{\mathbf{N}}=\left(N_{0}=2, N_{1}=36\right)^{[12]}$. For class-0 and class-1, the marginal probability $P_{m r}$, queueing length $L_{\text {system mr }}$ and the throughput $\lambda_{m r}$ can be calculated by the iteration algorithm presented in section 2.3. Table 2 shows the results and the percent errors of the analytical method and simulation.

\section{B. Analysis of the results}

Most items in Table 2 shows the accuracy of analytical method is relatively good and the approximations are reasonable.

1) Table2 shows most analytical method and simulation results for queueing length agree within $7 \%$. The queue lengthes of early warning aircrafts are nearly the same accurate as strickers.

2) The throughputs: The throughputs of early warning aircrafts are more accurate than strickers. The values of strickers' throughputs are slightly higher than that gained by simulation. However, the analytical results are still relatively accurate, taking the random of simulation into consideration.

3) The cycle time of early warning aircrafts 
is $\sum R_{\mathrm{Z}}=\mathrm{N}_{0} / \lambda_{0 i}(i=1)=3.09$ hours, while, strickers requires 5.25 hours to operate for a cycle.

4) The asymptotic sortie generation rate for early warning aircrafts is equal to $\lambda_{08}=0.6487$ sorties per hour and $\lambda_{18}=6.8618$ sortie per hour for strickers.

5) Figure2 shows the SGR for strickers rises to a peak and then falls down with the number of early warning aircrafts rising. When the number of early warning aircrafts is relatively large, the SGR of strickers falls down more rapidly.

In addition to analytical method and simulation results, Figure 2 displays analytical method and simulation results for utilizations. The utilizations for early warning aircrafts (Class 0 ) are small enough (the largest value is less than $12 \%)$. For strickers, the utilizations at repairing station $\left(\mathrm{C}_{3}\right)$ and maintenance station $\left(\mathrm{C}_{4}\right)$ are relatively high, while the utilizations at other stations are low. Thus, repairing and maintenance are the bottle neck of the whole system.

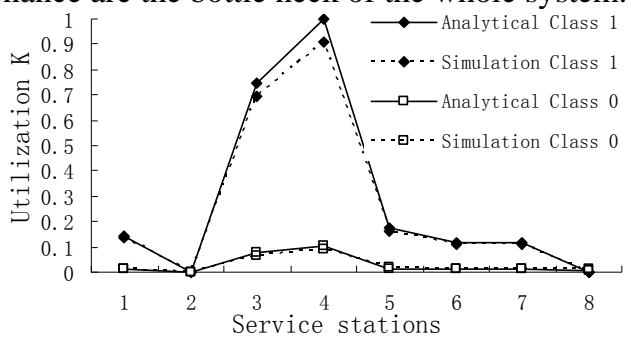

Fig.II Utilization of each service station

Table II Performance results for multi-class model

\begin{tabular}{|c|c|c|c|c|c|c|c|c|c|c|c|c|c|}
\hline \multirow{3}{*}{$\begin{array}{c}\text { Server } \\
\text { stations }\end{array}$} & \multirow{3}{*}{$\begin{array}{c}N_{0}=2 \\
N_{1}=36\end{array}$} & \multicolumn{6}{|c|}{ Queueing length $L_{\text {system }}$} & \multicolumn{6}{|c|}{ Throughput $\lambda(1 /$ hour) } \\
\hline & & \multicolumn{3}{|c|}{ Class 0} & \multicolumn{3}{|c|}{ Class 1} & \multicolumn{3}{|c|}{ Class 0} & \multicolumn{3}{|c|}{ Class 1} \\
\hline & & MVA & Simu & $\begin{array}{c}\text { Error } \\
\%\end{array}$ & MVA & Simu & $\begin{array}{c}\text { Error } \\
\%\end{array}$ & MVA & Simu & $\begin{array}{c}\text { Error } \\
\%\end{array}$ & MVA & Simu & $\begin{array}{c}\text { Error } \\
\%\end{array}$ \\
\hline 1 & Recovery & 0.0147 & 0.0164 & -10.21 & 0.1613 & 0.1640 & -1.60 & 0.6487 & 0.6899 & 5.98 & 6.8618 & 6.7106 & -2.25 \\
\hline 2 & Troubleshoot & 0.0097 & 0.0104 & -6.95 & 0.1029 & 0.0982 & 4.86 & 0.1946 & 0.2091 & 6.96 & 2.0585 & 1.9632 & -4.86 \\
\hline 3 & Repair & 0.3777 & 0.3605 & 4.80 & 4.9522 & 4.9295 & 0.46 & 0.1946 & 0.1849 & -5.25 & 2.0585 & 1.9389 & -6.17 \\
\hline 4 & Maintenance & 0.5363 & 0.5423 & -1.09 & 19.5471 & 20.0665 & -2.59 & 0.6487 & 0.6437 & -0.76 & 6.8618 & 6.5000 & -5.57 \\
\hline 5 & Turnaroud & 0.0649 & 0.0660 & -1.57 & 0.6874 & 0.6633 & 3.64 & 0.6487 & 0.6586 & 1.51 & 6.8618 & 6.6135 & -3.75 \\
\hline 6 & Catapult A & 0.0120 & 0.0122 & -1.23 & 0.1306 & 0.1270 & 2.94 & 0.3243 & 0.3288 & 1.38 & 3.4309 & 3.3518 & -2.36 \\
\hline 7 & Catapult B & 0.0120 & 0.0125 & -3.78 & 0.1306 & 0.1226 & 6.57 & 0.3243 & 0.3431 & 5.48 & 3.4309 & 3.2606 & -5.22 \\
\hline 8 & Sortie & 0.9725 & 0.9798 & -0.74 & 10.2875 & 9.8290 & 4.66 & 0.6487 & 0.6535 & 0.75 & 6.8618 & 6.5559 & -4.66 \\
\hline
\end{tabular}

\section{CONCLUSION}

To evaluate the sortie generation rate of carrier-borne aircrafts with different priorities, the sortie process is modeled as a closed network of multi-class multi-server nonpreemptive. The approximate analytical method in this paper offers an efficient way to obtain the mean performances of multi-class multi-server nonpreemptive closed queueing network. In just 0.5 second, results can be gained that would require more than 10 hours of running time in a simulation model. Simulation method provides results that shows the accuracy of analytical method is acceptable. It therefore can be a useful tool for calculating SGR of carrier-borne aircrafts.

\section{ACKNOWLEDGMENT}

This work was performed by members of Naval ships and system engineering Lab. Many thanks to students and professors that aided in the development of this program.

\section{REFERENCE}

[1] Dietz D C, Jerkins R C. Analysis of Aircraft Sortie with the use of a fork-join queueing network model[J]. Naval Logistics Research,1997,2(44):153-164.

[2] XIA Cuoqing, CHEN Hongzhao, WANG Yuanhui. Analysis of aircraft sortie generation rate based on closed queueing network model[J]. Journal of System Engineering ,2011,26(5):686-693(in Chinese).

[3] James W. Harris J. The Sortie Generation Rate Model[C]//The 2002 Winter Simulation Conference, USA: Simulation conference, 2002:864-868.

[4] Priya N W, Anura P J, Duane C B. A Nonpreemptive Priority Delay Model with Modified-Vacation Intervals for Homogeneous FDDI Networks[C]//Proceedings IEEE INFOCOM’95,USA: Local Computer Networks,1995:1282-1289.

[5] Chandramouli B H. MultiClass MultiServer Closed Priority Queueing Networks with Preemptive Resume[C]//Computer Perfermance Evalution CMPE230,USA: CMPE,2002:1-12.

[6] Bryant R M, Krzesinski A E, Lakshmi M S. The MVA Priority Approximation[J]ACM Transactions on Computer Systems,1984,2(4):335-359

[7] Akyildiz I F, Gunter B. Mean Value Analysis Approximation for Multiple Server Queueing Networks[J]. Performance Evaluation, 1988,2(8):77-91.

[8] Reiser M. A Queueing Network Analysis of Computer Communication Networks with Window Flow Control[J].IEEE Trans.Commun,1979,27(8):1199-1209.

[9] LU Chuanlai. Theory of Queues[M].Beijing: Beijing university of posts and technology press,1993.6:175-176 (in Chinese).

[10]Baynat B, Dallery Y.A Product-form Approximation Method for General Closed Queueing Networks with Several Classes of Customerers[J].Performance Evaluation,1996,24(22): 165-188. 